\title{
A FAMILY OF WINDSCHITL TYPE APPROXIMATIONS FOR GAMMA FUNCTION
}

\section{ZHEN-HANG YANG AND JING-FENG TIAN}

Abstract. In this paper, we present a family of high accurate approximation formulas

$$
\mathscr{W}_{p}(x)=\sqrt{2 \pi x}\left(\frac{x}{e}\right)^{x}\left(x \sinh \frac{1}{x}\right)^{x / 2} \exp \left(\frac{1}{1620 x^{5}} \frac{x^{2}+p}{x^{2}+p+33 / 35}\right)
$$

for gamma function $\Gamma(x+1)$ with parameter $p \geqslant-33 / 35$, and prove the function

$$
x \mapsto \ln \Gamma(x+1)-\ln \mathscr{W}_{p}(x)
$$

is strictly increasing and concave on $(0, \infty)$ if and only if $p \geqslant 158 / 315$. This yields some new sharp approximations for gamma function.

Mathematics subject classification (2010): Primary 33B15, 26D15, Secondary 26A48, 26 A51.

Keywords and phrases: Gamma function, monotonicity, convexity, Windschitl type approximation.

\section{REFERENCES}

[1] W. BURnside, A rapidly convergent series for $\log N$ !, Messenger Math. 46 (1917), 157-159.

[2] R. W. GosPeR, Decision procedure for indefinite hypergeometric summation, Proc. Natl. Acad. Sci. USA, 75 (1978), 40-42.

[3] N. BatiR, Sharp inequalities for factorial n!, Proyecciones 27, 1 (2008), 97-102.

[4] C. Mortici, On the generalized Stirling formula, Creative Math. Inf. 19, 1 (2010), 53-56.

[5] S. Ramanujan, The Lost Notebook and Other Unpublished Papers, Springer, Berlin, 1988.

[6] W. D. SмIтH, The gamma function revisited, http://schule. bayernport. com/gamma/gamma05.pdf, 2006.

[7] http://www.rskey.org/gamma.htm.

[8] G. NemES, New asymptotic expansion for the Gamma function, Arch. Math. (Basel), 95 (2010), 161169.

[9] C. MoRTiCi, Improved asymptotic formulas for the gamma function, Comput. Math. Appl. 61 (2011), 3364-3369.

[10] C. MorTici, A new fast asymptotic series for the gamma function, Ramanujan J. 38, 1 (2015), 549559.

[11] ZH.-H. YAng AND Y.-M. ChU, Asymptotic formulas for gamma function with applications, Appl. Math. Comput. 270 (2015), 665-680.

[12] CH.-P. Chen, Asymptotic expansions of the gamma function related to Windschitl's formula, Appl. Math. Comput. 245 (2014), 174-180.

[13] CH.-P. CHEN, A more accurate approximation for the gamma function, J. Number Theory 164 (2016), 417-428.

[14] D. LU, A new sharp approximation for the Gamma function related to Burnside's formula, Ramanujan J. 35, 1 (2014), 121-129.

[15] D. Lu, L. Song AND C. MA, Some new asymptotic approximations of the gamma function based on Nemes' formula, Ramanujan's formula and Burnside's formula, Appl. Math. Comput. 253 (2015), $1-7$.

[16] Zh. YAng And J.-F. Tian, A comparison theorem for two divided differences and applications to special functions, J. Math. Anal. Appl. 464 (2018), 580-595. 
[17] N. BATIR, Inequalities for the gamma function, Arch. Math. 91 (2008), 554-563.

[18] C. MORTICI, An ultimate extremely accurate formula for approximation of the factorial function, Arch. Math. 93, 1 (2009), 37-45.

[19] C. MoRTICI, New sharp inequalities for approximating the factorial function and the digamma functions, Miskolc Math. Notes 11, 1 (2010), 79-86.

[20] J.-L. ZHAO, B.-N. GUO AND F. QI, A refinement of a double inequality for the gamma function, Publ. Math. Debrecen 80, 3-4 (2012), 333-342.

[21] C. MorTicI, Further improvements of some double inequalities for bounding the gamma function, Math. Comput. Model. 57 (2013), 1360-1363.

[22] F. QI, Integral representations and complete monotonicity related to the remainder of Burnside's formula for the gamma function, J. Comput. Appl. Math. 268 (2014), 155-167.

[23] ZH.-H. YANG AND J. Tian, Monotonicity and sharp inequalities related to gamma function, J. Math. Inequal. 12, 1 (2018), 1-22.

[24] H. Alzer, On some inequalities for the gamma and psi functions, Math. Comput. 66, 217 (1997), 373-389.

[25] ZH.-H. YANG, Approximations for certain hyperbolic functions by partial sums of their Taylor series and completely monotonic functions related to gamma function, J. Math. Anal. Appl. 441 (2016), 549-564.

[26] ZH.-H. YANG AND J.-F. TIAN, Two asymptotic expansions for gamma function developed by Windschitl's formula, Open Math. 16 (2018), 1048-1060.

[27] C.-P. CHEN AND R. B. PARIS, Inequalities, asymptotic expansions and completely monotonic functions related to the gamma function, Appl. Math. Comput. 250 (2015), 514-529.

[28] D. LU, L. Song AND C. MA, A generated approximation of the gamma function related to Windschitl's formula, J. Number Theory, 140 (2014), 215-225.

[29] H. AlZeR, Sharp upper and lower bounds for the gamma function, Proc. R. Soc. Edinb. 139A (2009), 709-718.

[30] ZH.-H. YANG AND J.-F. TIAN, An accurate approximation formula for gamma function, J. Inequal. Appl. 2018 (2018): 56.

[31] J. Tian, W. Wang, W.-S. Cheung, Periodic boundary value problems for first-order impulsive difference equations with time delay, Adv. Differ. Equ. 2018 (2018): 79.

[32] J.-F. TIAn, Triple Diamond-Alpha integral and Hölder-type inequalities, J. Inequal. Appl. 2018 (2018): 111.

[33] J.-F. TIAN, M.-H. HA AND CH. WANG, Improvements of generalized Hölder's inequalities and their applications, J. Math. Inequal. 12, 2 (2018), 459-471.

[34] M. AbramowttZ AND I. A. STEGUn, Handbook of Mathematical Functions with Formulas, Graphs and Mathematical Tables, Dover, New York, 1972. 